\title{
Multi-agent system based on self-adaptive differential evolution for solving dynamic optimization problems
}

\author{
Aleš Čep \\ Faculty of Electrical Engineering and Computer \\ Science \\ University of Maribor \\ ales.cep@um.si
}

\author{
Iztok Fister \\ Faculty of Electrical Engineering and Computer \\ Science \\ University of Maribor \\ iztok.fister@um.si
}

\begin{abstract}
This article presents the multi-agent system based on a selfadaptive differential evolution algorithm for solving dynamic problems. Characteristics of dynamic problems are that objective function, with which the quality of solutions is evaluated, changes over time. These changes can occur either after some predefined number of generations or, more commonly, in each generation, where the online responses are desired by the evolutionary algorithms. In this study, the former kind of problems were taken into consideration and were solved using multi-agent systems. Each agent in the systems is implemented as a self-adapted differential evolution with constant population size. In our comparative study, multiagent systems consisting of various population sizes in combination with various number of agents were compared, by solving the benchmark functions provided for CEC'09 Competition on Dynamic Optimization with respect to the same number of the fitness function evaluation. The main obstacle when using smaller populations is to prevent the stagnation of the population. That was partially overcome by using additional mechanisms such as: diversity measurement and information sharing between agents. As a result, the most appropriate multi-agent system was searched for, and the results of the best found multi-agent system were compared with the state-of-the-art algorithms.
\end{abstract}

\section{Keywords}

Differential evolution, self-adaptive differential evolution, multi-agent system, dynamic problems

\section{INTRODUCTION}

Many of real-world problems are dynamic in their nature. Dynamic optimization problems (DOP) [4] can be described as problems, where decision maker has to make multiple decisions over time and the overall performance depends on all decisions made in that time. Decisions are made sequentially over time, but it depends on the type of problems, if this decisions are made by time triggered (periodic) or event triggered events. Consequently, the value of a fitness function that is optimal at some time, is not necessary optimal at another and vice versa.

Differential evolution (DE) was proposed by Storn and Price in [11] and is a powerful evolutionary algorithm (EA) frequently used for solving global optimization problems. Its simplicity and effectiveness have been proven in many realworld applications. DE emulates natural evolution process using three operators: mutation, crossover and selection. Brest et al [1] proposed jDE algorithm which extends the original DE algorithm with self-adaptation of DE control parameters, i.e., the scale rate and the frequency of crossover rate. This self-adaptation is performed before generating the trial vector and therefore it influences creation of new trial vectors. These controls parameters are added to a representation of individuals and undergo operation of variation operators (i.e., mutation and crossover) during the evolutionary cycle.

Morrison [8] presented new EA architecture for solving DOP where he uses so-called sentinels, i.e., individuals in a population that are spread across search space whose location does not change. In this way they can be used for detecting changes of environment. Brest et al [2] presented multipopulation $\mathrm{jDE}\left(\mathrm{jDE}^{*}\right)$ algorithm with the ageing mechanism and the use of archive where they stored the current best individuals after the environment change was detected. There was no information sharing between individuals. Yang et al [13] proposed an algorithm for improving DE with population adaptation approach, where it calculates the standard deviation of individuals' in $j$-th dimension at generation $G$. Halder et al [5] presented an algorithm CDDE_Ar that uses a multi-population method where clusters (sub-populations) are partitioned according to the spatial locations of trial solutions. During the evolution process, population is periodically divided in different number of clusters which causes certain information sharing during the optimization process. Lepagnot et al [6] presented MLSDO algorithm, which is based on several coordinated local searches and on the archiving of the found local optima, in order to track them after a change in the objective function. Novoa et al [9] proposed an algorithm mSQDEi, a multi-population algorithm with self-adaptive strategy for controlling the population diversity and an interaction mechanism between individuals.

This paper proposes multi-agent system based on self-ada- 
ptive differential evolution (MAS-jDE) for solving DOP. These are a class of problems, where the environment changes over time. Autonomous agents that are capable of detecting these changes on their own, try to solve DOP by using jDE and maintaining various sized populations. An evolutionary progress can typically stagnate when using agents with smaller populations. Therefore, we tried to prevail this problem by implementing two mechanism: detection of stagnation by calculating population's diversity and migration of individuals between agents. Experiments with various configurations of multi-agent systems were conducted, where the number of agents and the population sizes were varied. Indeed, these both parameters were selected such that the algorithms in each configurations spent the same number of the fitness function evaluations. In this way, fair comparison between different MAS-jDE configurations can be expected. Additionally, the performance of the proposed algorithm MAS-jDE was also compared to the others state-of-the-art algorithms for solving DOP.

The remainder of the paper is organized in the following way. Section 2 describes the DOPs. Section 3 describes the original DE, its extension jDE and the proposed MAS-jDE algorithm. Section 4 illustrates the results of experiments that were conducted and provides discussion on them. Finally, Section 5 concludes this paper with summarizing the performed work and outlining directions for the future work.

\section{PROBLEM DEFINITION}

This paper is devoted to solving DOPs. Li et al. in [7] defined these kind of problems as follows:

$$
\mathrm{DOP}=f(\mathbf{x}, \phi, t),
$$

where DOP is the dynamic optimization problem, $f$ is an evaluation function, $x$ is a feasible solution in the solution set $\mathrm{X}, \phi$ (so called change type) is a system control parameter determining the solution's distribution in the fitness landscape and $t$ is real-world time. Changes in environment can be results of a change of system control parameters describing environment state or a change in evaluation function. These changes can occur either after some predefined number of generations or, more commonly, in each generation, where the online responses are desired by the evolutionary algorithms.

The goal of the algorithm for solving DOP is to detect the change of environment and find the new optimum. Problem frequently occurring in evolutionary algorithms for DOP is a stagnation of population. Evolutionary process moves population to or very near to the local or global optimum, which causes a very small diversity of the population. When it comes to environmental change, the population with small diversity cannot explore search space efficiently enough. Usually this could mean, that the algorithm will not be able to find the next global optimum.

\section{THE PROPOSED ALGORITHM}

The proposed MAS-jDE algorithm combines a knowledge from two domains: evolutionary algorithms (EAs) [3] and multi-agent systems (MAS) [12]. In line with this, three algorithm are described at first, i.e., differential evolution (DE), followed by self-adaptation differential evolution (jDE) that is an extension of DE. Finally, the proposed MAS-jDE is presented from the multi-agent aspect.

\subsection{Differential evolution}

DE [11] is a well established EA, developed by Storn and Price [11]. It is a population-based algorithm, whose population is defined as a set of real-valued vectors representing different solutions of the problem:

$$
\mathbf{x}_{\mathbf{i}}=\left\{x_{i, 1}, x_{i, 2}, \ldots, x_{i, D}\right\},
$$

for $i=1, \ldots, N P$, where $N P$ represents the population size and $D$ is the dimensionality of the problem. Like in other EAs, the first step in DE is a random initialization of the population. Until the termination condition is satisfied, the population undergoes operation of three evolutionary operators by creating trial vectors: mutation, crossover and selection.

Mutation creates mutant vector $v_{i}^{(G+1)}$ from parent's population. Different mutation DE strategies have been used in literature $[10,11]$, but in original article [11] authors used the so-called 'DE/rand/1/bin':

$$
v_{i}^{(G+1)}=x_{r 0}^{(G)}+F \cdot\left(x_{r 1}^{(G)}-x_{r 2}^{(G)}\right),
$$

where $r 0, r 1$ and $r 2$ represent random integer numbers in range $[1, N P]$, where it holds $r 0 \neq r 1 \neq r 2$, and the scale factor is a real number in range $F \in[0,2]$.

Crossover operator introduces a little diversity in the population of solutions. It creates a trial vector $u_{i, j}^{(G+1)}$ from a combination of elements from either the mutation vector or the corresponding parent vector as:

$$
u_{i, j}^{(G+1)}= \begin{cases}v_{i, j}^{(G+1)} & \text { if } \operatorname{rand}(0,1) \leq C R \text { or } j=j_{\text {rand }}, \\ x_{i, j}^{(G)} & \text { otherwise, }\end{cases}
$$

where the crossover rate is defined in range $C R \in[0,1)$ and determines a probability of creating a trial vector from the mutation vector. Index $j_{\text {rand }}$ is randomly selected integer defined in range $[1, D]$ that is used to ensure that the algorithm makes at least one change in trial vector regarding previous generation.

Selection compares the results of fitness function from newly created trial vector $u_{i, j}^{(G+1)}$ and parent vector $x_{i, j}^{(G)}$. If the problem is minimization problem, it is defined as follows:

$$
x_{i}^{(G+1)}= \begin{cases}u_{i}^{(G+1)} & \text { if } f\left(u_{i}^{(G+1)}\right) \leq f\left(x_{i}^{(G)}\right) \\ x_{i}^{(G)} & \text { otherwise. }\end{cases}
$$

The fitness function depends on the problem to be solved.

\subsection{Self-adaptive DE}

Brest et al. [1] presented self-adaptive differential evolution algorithm (jDE), where control parameters $F_{i}$ and $C R_{i}$ are added to representation of individuals and adapted during the evolution process using following equations:

$$
\begin{gathered}
F_{i}^{(G+1)}= \begin{cases}F_{l}+\operatorname{rand}_{1} \cdot F_{u} & \text { if } \operatorname{rand}_{2}<\tau_{1}, \\
F_{i}^{(G)} & \text { otherwise, }\end{cases} \\
C R_{i}^{(G+1)}= \begin{cases}\operatorname{rand}_{3}, & \text { if } \operatorname{rand}_{4}<\tau_{2}, \\
C R_{i}^{(G)} & \text { otherwise, }\end{cases}
\end{gathered}
$$


where $\operatorname{rand}_{j}, j \in[1-4]$ represents random number in range $[0,1]$ and $F_{i}^{(G+1)}$ is in range $[0.1,1]$. Constants $\tau_{1}$ and $\tau_{2}$ are so-called learning rates determining the frequency of updating control parameters $F$ and $C R$, and were set to 0.1 .

Adaptation of control parameters according to Eq. (6)-(7) is performed after 10 generations in average, and therefore has a big influence on mutation and crossover operators.

\subsection{Multi-agent system based on jDE for solv- ing DOPs}

Multi-agent system based on jDE for solving DOPs (MASjDE) implements system of multiple autonomous agents, that run autonomously based on jDE algorithm using separated populations of the same size. Multi-agent systems are systems composed of multiple interfacing intelligent agents. Each agent is a computationally entity like an algorithm that is placed in an environment in order to achieve its objectives [12]. In our case, each agent is devoted to search for a best solution in own region of the whole search space. Using the interaction between agents, they are able to enrich their limited knowledge with informations obtained from other agents in the MAS.

There are four main issues that must be solved when developing the algorithm: agent's population size determination, environment change detection, maintaining the population diversity and information exchange between agents. According to our assumption of fairness, it holds that if system contains more agents, the population size of each agent is decreased. This fact can cause a stagnation problem, where algorithm gets stuck in a local optimum and can not get out, due to the lower population diversity. To avoid this problem, we introduce mechanism for measuring the population diversity that uses the following principle: When the diversity of population is insignificant, the MAS-jDE begins migration of individuals between agents. The pseudo-code of the algorithm is illustrated in Algorithm 1.

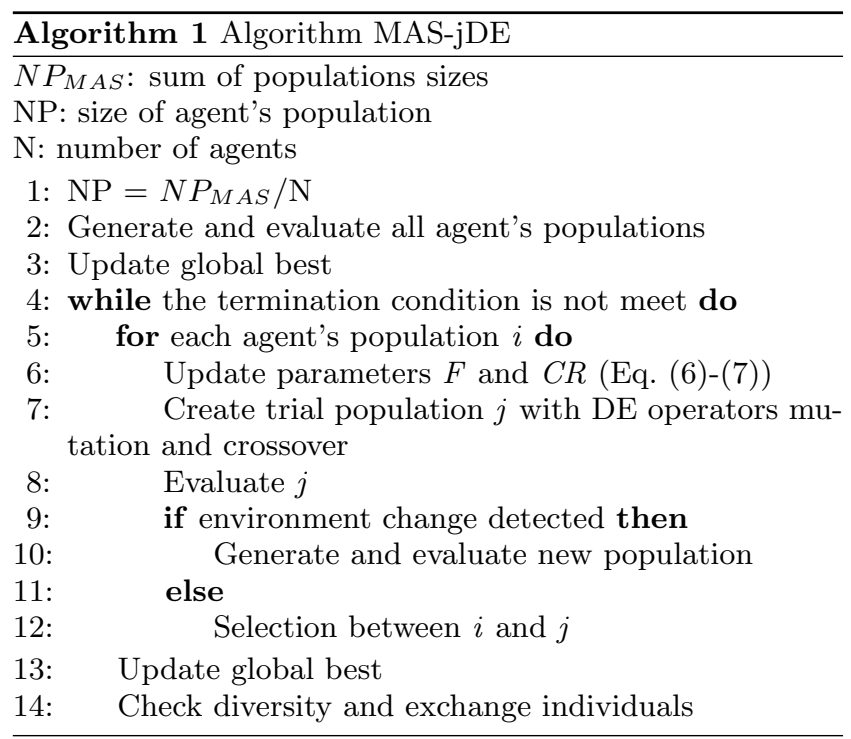

In the remainder of the paper, the exposed issues of the proposed MAS-jDE algorithm are discussed in details.

\subsubsection{Agent's population size determination}

In order to ensure that the comparison between different MAS is as fair as possible, we introduce the total population size $N P_{M A S}$, that determines the number of vectors in the so-called grand population. Obviously, the MAS-jDE with grand population is equivalent to the original $\mathrm{jDE}$ using a single population. In MAS-jDE, the members of this grand population are divided among agents evenly. The higher the number of agents in the MAS, the smaller is their population size. In line with this, the agent's population size $N P$ is obtained as:

$$
N P=\frac{N P_{M A S}}{N},
$$

where $N$ represents the number of agents. Because all three mentioned variables have to be integers, not all combinations of $N P_{M A S}$ and $N$ are feasible.

\subsubsection{Environment change detection}

An evolutionary cycle starts after a random initialization and evaluation of agent's population. The DE mutation and crossover operate on $N P-1$ trial vectors only, because the last vector is used as a sentinel [8] that never changes. Thus, the sentinel's fitness function value is changed with the change of the environment. Therefore, we first check after the newly generated population is evaluated, if the fitness value of sentinel distinguishes from the old value. In this case, we declare that the environment has changed, consequently reinitialize agent population randomly and evaluate the new fitness values for the whole population.

\subsubsection{Maintaining the population diversity}

The stagnation problem can be evident at the population as whole or at the component level [13]. In MAS-jDE, we detect stagnation by calculating agent's population diversity using the following equations $[8,13]$ :

$$
a_{j}^{(G)}=\frac{\sum_{i=1}^{N P-1} x_{i, j}^{(G)}}{N P-1}
$$

$$
\text { diversity }=\sqrt{\sum_{j=1}^{D} \sum_{i=1}^{N P-1}\left(x_{i, j}^{(G)}-a_{j}^{(G)}\right)^{2}}
$$

where $a_{j}^{(G)}$ stores the mean of population in $j$ th dimension. $j$ is in range $[1, D]$, where $D$ is a problem dimension. $\mathbf{x}_{i}$ is an individual in population.

Diversity in population is checked after each generation to detect stagnation as quickly as possible. If it falls bellow threshold level, migration between agents starts. Threshold level must be set high enough, so that agent has enough diversity in population to continue exploring search-space and low enough, so it can close-in to the optimum.

\subsubsection{Information exchange between agents}

When diversity in population falls bellow threshold, migration between agents starts (Algorithm 2). Idea is as follows. Each agent begins exploration in different part of search space and therefore it's search is limited to the specific region. When new vectors are migrated from another agent, it is expected that the diversity of population will increase. Thereby, the agent will be attracted to explore the new promising regions in the search space. Procedure 
of sharing information between agents is described in three steps, as follows:

1. step: The best vectors from all agents are found.

2. step: From these vectors, find the one that is the most diverse from the best vector in current agent based on Euclidian distance (Eq. (11)).

3. step: Using Euclidian distance (Eq. (11)), find two vectors that are closest to each other in the current agent (Algorithm 3) and replace the worse based on fitness function result, with the vector found is step 2 .

Distance between vectors is calculated as Euclidean distance:

$$
d(p, q)=\sqrt{\sum_{j=1}^{D}\left(p_{j}-q_{j}\right)^{2}},
$$

where $p$ and $q$ represent $D$ dimensional vector.
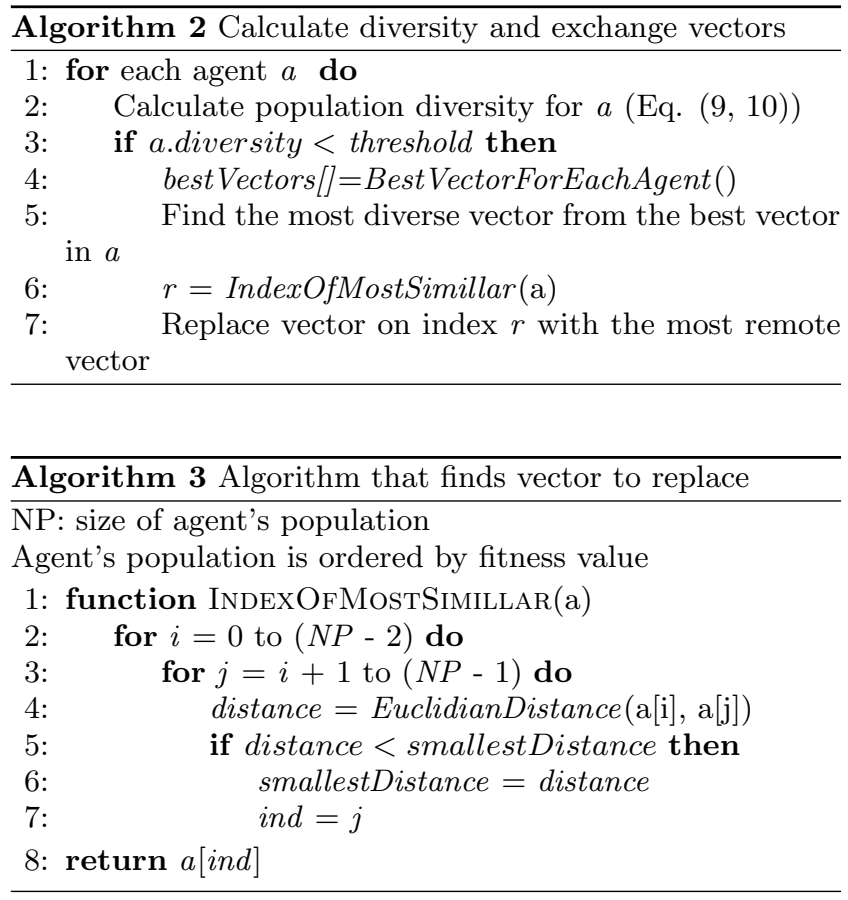

\section{EXPERIMENT AND RESULTS}

The goal of our experimental work was twofold. Firstly to test the MAS-jDE with different parameters settings, and thus find the best settings for parameters: the population size $N P$ and the number of agents $N$. Secondly to compare the results obtained by the MAS-jDE with the results of the other state-of-the-art algorithms. In both experiments, all algorithms solved CEC'09 benchmark test functions for DOP.

The algorithm MAS-jDE used the following parameters during experiments:

- the total population size was varied as $N P_{M A S} \in\{50$, $100,200,400\}$
- the number of agents was set to values $N \in\{1,5,8,10$, $16,20,25\}$, from which only the feasible (Table 1) were considered,

- the scale size was initially drawn randomly from uniform distribution in interval $F_{i}^{(0)} \in[0,1]$,

- the crossover probability was initially drawn randomly from uniform distribution in interval $C R_{i}^{(0)} \in[0,1]$,

- the diversity threshold in Algorithm 2 was set to threshold $=1$.

\subsection{Dynamic optimization benchmark}

Algorithms in the study were tested on benchmark test suite provided by CEC'09 organizers for Competition on Dynamic Optimization [7], which uses the generalized dynamic benchmark generator. Benchmark defines seven change types: small-step change $\left(T_{1}\right)$, large-step change $\left(T_{2}\right)$, random change $\left(T_{3}\right)$, chaotic change $\left(T_{4}\right)$, recurrent change $\left(T_{5}\right)$, recurrent change with noise $\left(T_{6}\right)$, and random change with changed dimension $\left(T_{7}\right)$.

There are six test functions defined in the real-space:

- $F_{1}$ : Rotation peak function (with 10 and 50 peaks)

- $F_{2}$ : Composition of Sphere's function

- $F_{3}$ : Composition of Rastrigin's function

- $F_{4}$ : Composition of Griewank's function

- $F_{5}$ : Composition of Ackley's function

- $F_{6}$ : Hybrid Composition function

In summary, there are 49 specific test cases and each test case contains 60 changes. Test cases run independently 20 times to obtain the mark on specific case. Overall performance perf is the sum of the performance measures obtained for each function and each change type as follows:

$$
\text { perf }=\sum_{i=1}^{49} \operatorname{mark}_{i} \cdot 100,
$$

where the performance measure mark $_{i}$ is defined as follows:

$$
\operatorname{mark}_{i}=\frac{w_{i}}{R \cdot C} \sum_{l=1}^{R} \sum_{j=1}^{C} r_{l j},
$$

where $w_{i}$ denotes weight of the problem $i$ (see [7] for more details), $\mathrm{R}$ the number of runs and $\mathrm{C}$ is the number of changes (in our case $R=20$ and $C=60$ ). The variable $r_{l j}$ is expressed as:

$$
r_{l j}=r_{l j}^{l a s t} /\left(1+\sum_{s=1}^{S}\left(1-r_{l j}^{s}\right) / S\right),
$$

where $r_{l j}^{l a s t}$ denotes the relative ratio between fitness value of best individual and global optimum of the $j$-th environment, $r_{l j}^{s}$ the relative ratio between fitness value of best individual and global optimum at the $s$-th sampling during one change, and $S$ is total number of samples for each environment. 


\subsection{Computer configuration}

The computer used for experimental work had the following configuration:

- System: Windows 10

- CPU: Intel Core i7-4790

- RAM: 16 GB

- Programming language: $\mathrm{C}++$

\subsection{Results}

As mentioned in Section 3.3.1, not all combinations of parameters $N P_{M A S}$ and $N$ are feasible. Therefore, in the first experiment, the following feasible combinations as presented in Table 1 were used in our study.

Table 1: Feasible combinations of agent's number $N$.

\begin{tabular}{|r|r|r|r|r|r|r|r|}
\hline \multirow{2}{*}{$N P_{M A S}$} & \multicolumn{7}{|c|}{$N$} \\
\cline { 2 - 8 } & \multicolumn{1}{|c|}{1} & \multicolumn{1}{|c|}{8} & 10 & \multicolumn{1}{|c|}{16} & 20 & 25 \\
\hline 50 & 50 & 10 & $\mathbf{6 . 2 5}$ & 5 & $\mathbf{3 . 1 2}$ & $\mathbf{2 . 5}$ & $\mathbf{2}$ \\
100 & 100 & 20 & $\mathbf{1 2 . 5}$ & 10 & $\mathbf{6 . 2 5}$ & 5 & 4 \\
200 & 200 & 40 & 25 & 20 & $\mathbf{1 2 . 5}$ & 10 & 8 \\
400 & 400 & 80 & 50 & 40 & 25 & 20 & 16 \\
\hline
\end{tabular}

Values in the table present agent's population size $N P$ and must be integer. When the results of division of $N P_{M A S}$ by $N$ is not an integer value, this combination of parameters is declared as infeasible. The infeasible values are presented bold in the table. Value in row $N P_{M A S}=50$ and column $N=25$ is also infeasible, because mutation strategy 'DE/rand/1/bin' in DE (Eq. (3)) requires at least 4 individuals in population.

Table 2 shows the overall performance perf (Eq. (12)) obtained in the first experiment by the MAS-jDE algorithm for all combinations of $N P_{M A S}$ and $N$. Higher values in table present the better results. Non-valid combinations of parameters are marked with " $\mathrm{n} / \mathrm{a}$ " in the table. Thus, the last row depicts the average overall performance values according to the number of agents $(N)$, where the infeasible values of population sizes were ignored.

The highest performance value perf $=70.1968$ was achieved, when the grand population size was $N P_{M A S}=200$ and the MAS consisted of $N=10$ agents. The last row with average performance results shows, that the MAS-jDE consisting of 8 agents returned the best results. However, this result was based only on two instances. Obviously, when all four overall performance values are taken into consideration, the MASjde using 5 agents has shown to be the best.

The results in Table 2 also show the stagnation effect in algorithm, although both features, i.e., the diversity measurements and migration of individuals were activated. Consequently, the worst results were achieved, when the grand population was divided between large number of agents. Performance also worsened, when the number of agents in the MAS with the single population increased.

Results from Table 2 are illustrated in Figure 1, comparing the multi-agent systems of different total population sizes

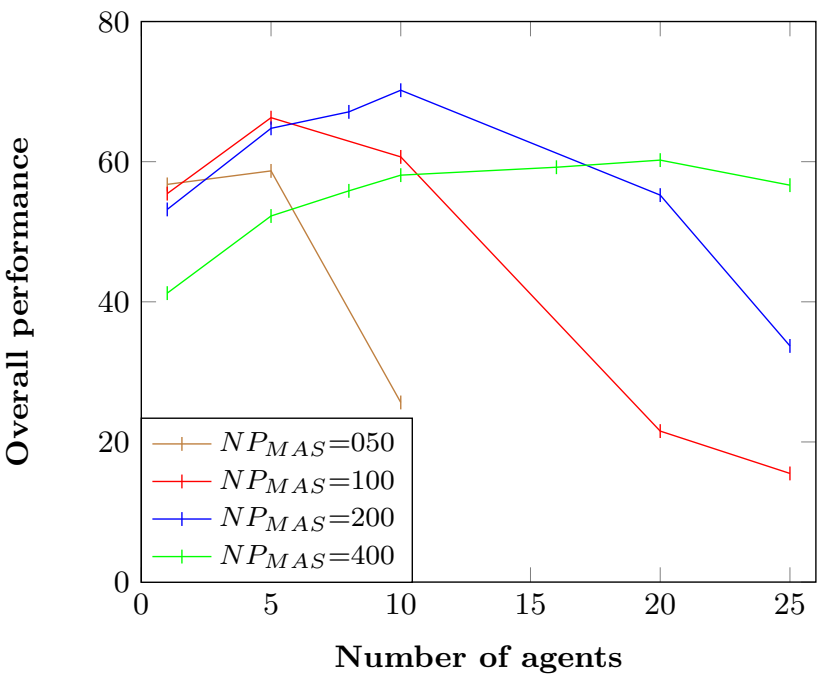

Figure 1: Comparison of various population sizes

and different number of agents according to overall performance results of CEC'09 benchmark. It can be observed that the best result of MAS-jDE was obtained by the MAS with the total population size of $N P_{M A S}=200$. Thus, it is evident that the total population size of $N P_{M A S}=50$ is inappropriate for building MAS, because of insufficient population size in agent. In general, the MASs with higher total population sizes are more convenient for achieving the best results.

Table 3 presents more detailed results for the best solution of MAS-jDE, with $N=10$ agents and the total population size of $N P_{M A S}=200$. The best results were obtained by solving the Ackley's function $\left(F_{5}\right)$ for all 7 change types. Good results were achieved for all functions at small-step $\left(T_{1}\right)$ and chaotic $\left(T_{4}\right)$ change. Algorithm performed very well for functions $F_{2}$ and $F_{4}$ for recurrent change with noise $\left(T_{6}\right)$ and for function $F_{6}$ with random change $\left(T_{3}\right)$, recurrent change $\left(T_{5}\right)$ and recurrent change with noise $\left(T_{6}\right)$.

In the second experiment, the solution with highest overall performance perf (Eq. (12)) found in the first experiment, was compared to the DOP DE, DOP jDE (i.e., equivalent to single agent MAS-jDE) and the following stateof-the-art algorithms: jDE* [2], CDDE_Ar [5], MLSDO [6] and mSQDE-i [9]. Algorithms DOP DE and DOP jDE are original DE [11] and jDE [1] algorithms that were adopted to solve DOP, by randomly reinitializing population when change in environment is detected. The results for algorithms DOP DE and DOP jDE were contributed using our own implementations of the algorithms, while the results for others were obtained from their respective literature.

The comparative study of mentioned algorithms was made according to the overall performance values perf for 49 test cases of CEC'09 benchmark functions. The results are depicted in Table 4 and show that the MAS-jDE algorithm achieved better results than the DOP DE, DOP jDE, jDE* and CDDE_Ar algorithms, while the mSQDE-i and MLSDO 
Table 2: Overall performance by total population sizes and number of agents

\begin{tabular}{|r|c|r|r|r|r|r|r|}
\hline \multirow{2}{*}{$N P_{\text {MAS }}$} & \multicolumn{7}{|c|}{$N$} \\
\cline { 2 - 8 } & 1 & \multicolumn{1}{|c|}{5} & \multicolumn{1}{c|}{10} & \multicolumn{1}{c|}{16} & \multicolumn{1}{c|}{20} & \multicolumn{1}{c|}{ n/a } \\
\hline 50 & 56.7574 & 58.6826 & n/a & 25.6366 & n/a & n/a & n/a \\
100 & 55.4424 & 66.2785 & n/a & 60.6823 & n/a & 21.5483 & 15.5059 \\
200 & 53.1856 & 64.7585 & 67.1079 & $\mathbf{7 0 . 1 9 6 8}$ & n/a & 55.2277 & 33.7031 \\
400 & 41.2382 & 52.2476 & 55.8367 & 58.0758 & 59.2057 & 60.2217 & 56.6458 \\
\hline Avg & 51.6559 & 60.4918 & 61.4723 & 53.6478 & 59.2057 & 45.6659 & 35.2849 \\
\hline
\end{tabular}

Table 3: Algorithm MAS-jDE overall performance for the best solution $\left(N P_{M A S}=200\right.$ and $\left.N=10\right)$

\begin{tabular}{|l|l|l|l|l|l|l|l|}
\hline & $F_{1}(10)$ & $F_{1}(50)$ & $F_{2}$ & $F_{3}$ & $F_{4}$ & $F_{5}$ & $F_{6}$ \\
\hline$T_{1}$ & 0.0149787 & 0.0148135 & 0.0183885 & 0.0152204 & 0.0190092 & 0.0220642 & 0.0152638 \\
\hline$T_{2}$ & 0.0141223 & 0.0137885 & 0.00997478 & 0.00335469 & 0.0108478 & 0.0227195 & 0.0156269 \\
\hline$T_{3}$ & 0.0137606 & 0.0129679 & 0.0145688 & 0.0065958 & 0.013584 & 0.02233 & 0.0170243 \\
\hline$T_{4}$ & 0.0149597 & 0.0149308 & 0.0142835 & 0.0112827 & 0.0178799 & 0.0229582 & 0.0135977 \\
\hline$T_{5}$ & 0.0141646 & 0.0146022 & 0.0115689 & 0.00435399 & 0.0114108 & 0.0225239 & 0.0177137 \\
\hline$T_{6}$ & 0.0136714 & 0.01301 & 0.0165458 & 0.0106462 & 0.0171642 & 0.0231245 & 0.0150692 \\
\hline$T_{7}$ & 0.0088615 & 0.00938833 & 0.0109534 & 0.00691136 & 0.0112994 & 0.0148324 & 0.0132556 \\
\hline Mark & 0.0945188 & 0.09350123 & 0.09628368 & 0.05836514 & 0.10119530 & 0.1505527 & 0.1075512 \\
\hline
\end{tabular}

algorithms outperformed it.

Table 4: Overall performance comparison

\begin{tabular}{|l|r|}
\hline Algorithm & Performance \\
\hline MAS-jDE & 70.1968 \\
DOP DE & 45.4996 \\
DOP jDE & 55.4424 \\
jDE* & 69.7269 \\
CDDE_Ar & 69.4700 \\
MLSDO & $\mathbf{8 1 . 2 7 6 0}$ \\
mSQDE-i & 77.1458 \\
\hline
\end{tabular}

\section{CONCLUSIONS}

In this paper we proposed MAS-jDE algorithm that is a MAS, where agents independently of each other explore the search space using the jDE. To prevent stagnation of the agent's populations, theirs population diversity is measured. If diversity falls bellow threshold, migration mechanism between agents takes place.

Algorithm was tested on CEC'09 benchmark functions for DOP. In line with this, two experiments were conducted. In the first, we compared multi-agent systems with different configurations, where the population sizes and the number of agents were varied. The results were compared according to the benchmark's overall performance measure. The best solution was found when the total population size was 200 and the number of agents was 10. From the results of the experiments we can observe, that although we used diversity measurements and migration of individuals, stagnation of population appears in combinations, where agent's had smaller population sizes.

The results of the MAS-jDE with the best parameter setting from the first experiment were also compared with other state-of-the-art optimization algorithms. The MAS-jDE showed much better results then DOP DE and DOP jDE.
Similar, but better results were achieved against jDE* and CDDE_Ar. Similarity between MAS-jDE and JDE* results can be contributed to the fact that both algorithms use the same self-adaptive algorithm to search for a global optimum. Our algorithm was outperformed by MLSDO and mSQDE-i.

Future plan is to study the results of diversity measurements and consequences of migrations of individuals more closely, and add mechanisms like k-means clustering algorithm to spread initial population more equally over search-space. Initial tests show promising results.

\section{REFERENCES}

[1] J. Brest, S. Greiner, B. Bošković, M. Mernik, and V. Žumer. Self-adapting control parameters in differential evolution: A comparative study on numerical benchmark problems. IEEE Transactions on Evolutionary Computation, 10(6):646-657, 2006.

[2] J. Brest, A. Zamuda, B. Bošković, M. S. Maučec, and V. Žumer. Dynamic optimization using self-adaptive differential evolution. In Evolutionary Computation, 2009. CEC'09. IEEE Congress on., pages 415-422. IEEE, May 2009.

[3] A. Eiben and J. E. Smith. Introduction to Evolutionary Computing. Natural Computing Series. Springer-Verlag Berlin Heidelberg, 1 edition, 2003.

[4] H. Fu, P. R. Lewis, B. Sendhoff, K. Tang, and X. Yao. What are dynamic optimization problems? In Evolutionary Computation (CEC), 2014 IEEE Congress on, 2014.

[5] U. Halder, S. Das, and D. Maity. A cluster-based differential evolution algorithm with external archive for optimization in dynamic environments. IEEE transactions on cybernetics, 43(3):881-897, June 2013.

[6] J. Lepagnot, A. Nakib, H. Oulhadj, and P. Siarry. A multiple local search algorithm for continuous dynamic optimization. Journal of Heuristics, 19(1):35-76, Feb. 2013. 
[7] C. Li, S. Yang, T. T. Nguyen, E. L. Yu, X. Yao, Y. Jin, H.-G. Beyer, and P. N. Suganthan. Benchmark generator for cec'2009 competition on dynamic optimization. Technical report, University of Leicester, University of Birmingham, Nanyang Technological University, 2008.

[8] R. W. Morrison. Designing Evolutionary Algorithms for Dynamic Environments. Springer-Verlag Berlin Heidelberg, 2004.

[9] P. Novoa-Hernández, C. C. Corona, and D. A. Pelta. Self-adaptive, multipopulation differential evolution in dynamic environments. Soft Computing, 17(10):1861-1881, Mar. 2013.

[10] K. V. Price, R. M. Storn, and J. A. Lampinen. Differential Evolution: A Practical Approach to Global Optimization, chapter The differential evolution algorithm, pages 37-134. Springer-Verlag Berlin Heidelberg, 2005.

[11] R. Storn and K. Price. Differential evolution - a simple and efficient heuristic for global optimization over continuous spaces. Journal of Global Optimization, 11(4):341-359, Dec. 1997.

[12] G. Weiss, editor. Multiagent Systems. MIT Press, 2nd edition, 2013.

[13] M. Yang, C. Li, Z. Cai, and J. Guan. Differential evolution with auto-enhanced population diversity. IEEE transactions on cybernetics, 45(2):302-315, 2015 . 